\title{
TRIANGULATED CATEGORIES WITHOUT MODELS
}

\author{
FERNANDO MURO, STEFAN SCHWEDE, AND NEIL STRICKLAND
}

\begin{abstract}
We exhibit examples of triangulated categories which are neither the stable category of a Frobenius category nor a full triangulated subcategory of the homotopy category of a stable model category. Even more drastically, our examples do not admit any non-trivial exact functors to or from these algebraic respectively topological triangulated categories.
\end{abstract}

Introduction. Triangulated categories are fundamental tools in both algebra and topology. In algebra they often arise as the stable category of a Frobenius category ([Hel68, 4.4], GM03, IV.3 Exercise 8]). In topology they usually appear as a full triangulated subcategory of the homotopy category of a Quillen stable model category Hov99, 7.1]. The triangulated categories which belong, up to exact equivalence, to one of these two families will be termed algebraic and topological, respectively. We borrow this terminology from [Kel06, 3.6] and [Sch06]. Algebraic triangulated categories are generally also topological, but there are many well-known examples of topological triangulated categories which are not algebraic.

In the present paper we exhibit examples of triangulated categories which are neither algebraic nor topological. As far as we know, these are the first examples of this kind. Even worse (or better, depending on the perspective), our examples do not even admit non-trivial exact functors to or from algebraic or topological triangulated categories. In that sense, the new examples are completely orthogonal to previously known triangulated categories.

Let $(R, \mathfrak{m})$ be a commutative local ring with $\mathfrak{m}=(2) \neq 0$ and $\mathfrak{m}^{2}=0$. Examples of this kind of rings are $R=\mathbb{Z} / 4$, or more generally $R=W_{2}(k)$ the 2-typical Witt vectors of length 2 over a perfect field $k$ of characteristic 2 . There are also examples which do not arise as Witt vectors, for instance the localization of the polynomial ring $\mathbb{Z} / 4[t]$ at the prime ideal $(2)$. We denote by $\mathcal{F}(R)$ the category of finitely generated free $R$-modules.

Theorem 1. The category $\mathcal{F}(R)$ has a unique structure of a triangulated category with identity translation functor and such that the diagram

$$
R \stackrel{2}{\longrightarrow} R \stackrel{2}{\longrightarrow} R \stackrel{2}{\longrightarrow} R
$$

is an exact triangle.

Given an object $X$ in an algebraic triangulated category $\mathcal{T}$ and an exact triangle

$$
A \stackrel{2 \cdot 1_{A}}{\longrightarrow} A \longrightarrow C \longrightarrow \Sigma A
$$

1991 Mathematics Subject Classification. 18E30, 55P42.

Key words and phrases. Triangulated category, stable model category.

The first author was partially supported by the Spanish Ministry of Education and Science under MEC-FEDER grants MTM2004-01865 and MTM2004-03629, the postdoctoral fellowship EX2004-0616, and a Juan de la Cierva research contract. 
the equation $2 \cdot 1_{C}=0$ holds, compare [Kel06, 3.6] and [Sch06. Since the ring $R$ satisfies $2 \cdot 1_{R} \neq 0$, the triangulation of the category $\mathcal{F}(R)$ is not algebraic. We cannot rule out the possibility of a topological model for $\mathcal{F}(R)$ as easily: the classical example of $A=S$ the sphere spectrum in the stable homotopy category shows that the morphism $2 \cdot 1_{C}$ can be nonzero in this more general context.

Nevertheless, $\mathcal{F}(R)$ is not topological either, which follows from Theorem2, Here we call an exact functor between triangulated categories trivial if it takes every object to a zero object.

Theorem 2. Every exact functor from $\mathcal{F}(R)$ to a topological triangulated category is trivial. Every exact functor from a topological triangulated category to $\mathcal{F}(R)$ is trivial.

Acknowledgements. We are grateful to Bernhard Keller for helpful conversations on the results of this paper, and to Amnon Neeman, who suggested the possibility of constructing a triangulated structure on $\mathcal{F}(\mathbb{Z} / 4)$ by using Heller's theory Hel68.

In the original version of this note the first author alone constructed the triangulation of the category $\mathcal{F}(\mathbb{Z} / 4)$ and proved that it does not admit any model. The second author joined the project later by providing a simpler and more general proof that the triangulation is not topological. The third author's contribution was an old preprint on the example considered in Remark 8 , which provided some guidance for the other results.

The triangulated categories. Let $\mathcal{T}$ be an additive category and let $\Sigma: \mathcal{T} \stackrel{\sim}{\rightarrow} \mathcal{T}$ be a self-equivalence that we call translation functor. A candidate triangle $(f, i, q)$ in $(\mathcal{T}, \Sigma)$ is a diagram

$$
A \stackrel{f}{\longrightarrow} B \stackrel{i}{\longrightarrow} C \stackrel{q}{\longrightarrow} \Sigma A,
$$

where $i f, q i$, and $(\Sigma f) q$ are zero morphisms. A morphism of candidate triangles $(\alpha, \beta, \gamma):(f, i, q) \rightarrow\left(f^{\prime}, i^{\prime}, q^{\prime}\right)$ is a commutative diagram

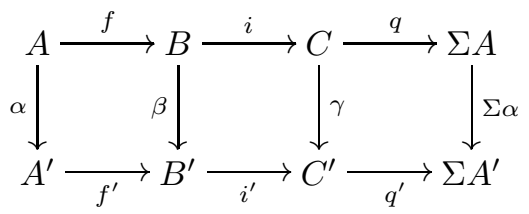

The category of candidate triangles is additive. The mapping cone of the morphism $(\alpha, \beta, \gamma)$ is the candidate triangle

$$
B \oplus A^{\prime} \stackrel{\left(\begin{array}{cc}
-i & 0 \\
\beta & f^{\prime}
\end{array}\right)}{\longrightarrow} C \oplus B^{\prime} \stackrel{\left(\begin{array}{cc}
-q & 0 \\
\gamma & i^{\prime}
\end{array}\right)}{\longrightarrow} \Sigma A \oplus C^{\prime} \stackrel{\left(\begin{array}{cc}
-\Sigma f & 0 \\
\Sigma \alpha & q^{\prime}
\end{array}\right)}{\longrightarrow} \Sigma B \oplus \Sigma A^{\prime}
$$

A homotopy $(\Theta, \Phi, \Psi)$ from $(\alpha, \beta, \gamma)$ to $\left(\alpha^{\prime}, \beta^{\prime}, \gamma^{\prime}\right)$ is given by morphisms

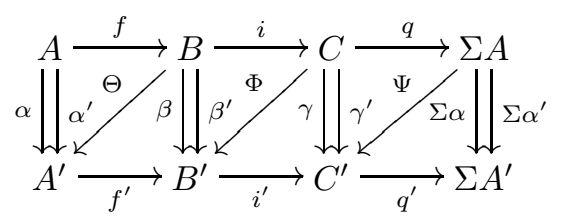

such that

$$
\beta^{\prime}-\beta=\Phi i+f^{\prime} \Theta, \quad \gamma^{\prime}-\gamma=\Psi q+i^{\prime} \Phi, \quad \Sigma\left(\alpha^{\prime}-\alpha\right)=\Sigma(\Theta f)+q^{\prime} \Psi .
$$


We say in this case that the morphisms are homotopic. The mapping cones of two homotopic morphisms are isomorphic. A contractible triangle is a candidate triangle such that the identity is homotopic to the zero morphism. A homotopy $(\Theta, \Phi, \Psi)$ from 0 to 1 is called a contracting homotopy. Any morphism from or to a contractible triangle is always homotopic to zero.

A triangulated category is a pair $(\mathcal{T}, \Sigma)$ as above together with a collection of candidate triangles, called distinguished or exact triangles, satisfying the following properties. The family of exact triangles is closed under isomorphisms. The candidate triangle

$$
A \stackrel{1}{\longrightarrow} A \longrightarrow 0 \longrightarrow \Sigma A,
$$

is exact. Any morphism $f: A \rightarrow B$ in $\mathcal{T}$ can be extended to an exact triangle like (3). A candidate triangle (3) is exact if and only if its translate

$$
B \stackrel{-i}{\longrightarrow} C \stackrel{-q}{\longrightarrow} \Sigma A \stackrel{-\Sigma f}{\longrightarrow} \Sigma B,
$$

is exact. Any commutative diagram

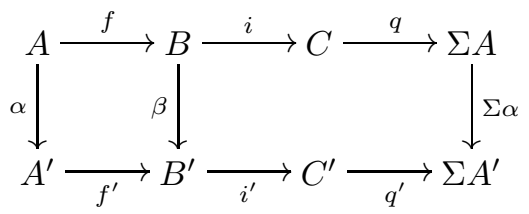

whose rows are exact triangles can be extended to a morphism whose mapping cone is also exact. This non-standard set of axioms for triangulated categories is equivalent to the classical one, see [Nee01, and works better for the purposes of this paper.

Now we are ready to prove Theorem 1 .

Proof of Theorem 1. Given an object $X$ in $\mathcal{F}(R)$ we consider the candidate triangle $X_{2}$ defined as

$$
X \stackrel{2}{\longrightarrow} X \stackrel{2}{\longrightarrow} X \stackrel{2}{\longrightarrow} X .
$$

We are going to prove that the category $\mathcal{F}(R)$ has a triangulated category structure with identity translation functor where the exact triangles are the candidate triangles isomorphic to the direct sum of a contractible triangle and a candidate triangle of the form (5).

The family of exact triangles is closed under isomorphisms by definition. The candidate triangle (4) is contractible, and hence exact. The ring $R$ is a quotient of a discrete valuation ring with maximal ideal generated by 2, see [Coh46, Corollary 3]; therefore any morphism $f: A \rightarrow B$ in $\mathcal{F}(R)$ can be decomposed up to isomorphism as

$$
f=\left(\begin{array}{lll}
1 & 0 & 0 \\
0 & 2 & 0 \\
0 & 0 & 0
\end{array}\right): A=W \oplus X \oplus Y \longrightarrow W \oplus X \oplus Z=B .
$$

Then $f$ is extended by the direct sum of (5) and the contractible triangle

$$
W \oplus Y \stackrel{\left(\begin{array}{ll}
1 & 0 \\
0 & 0
\end{array}\right)}{\longrightarrow} W \oplus Z \stackrel{\left(\begin{array}{ll}
0 & 0 \\
0 & 1
\end{array}\right)}{\longrightarrow} Y \oplus Z \stackrel{\left(\begin{array}{ll}
0 & 0 \\
1 & 0
\end{array}\right)}{\longrightarrow} W \oplus Y .
$$


The translate of a contractible triangle is also contractible, and the triangle (5) is invariant under translation. This proves that the translate of an exact triangle is exact. Translating a candidate triangle six times yields the original one, therefore if a candidate triangle has an exact translate then the original candidate triangle is also exact.

We say that a candidate triangle $A \stackrel{f}{\rightarrow} B \stackrel{i}{\rightarrow} C \stackrel{q}{\rightarrow} A$ is a quasi-exact triangle if

$$
A \stackrel{f}{\longrightarrow} B \stackrel{i}{\longrightarrow} C \stackrel{q}{\longrightarrow} A \stackrel{f}{\longrightarrow} B,
$$

is an exact sequence of $R$-modules. The exact triangles are all quasi-exact.

Now we are going to show that any diagram of candidate triangles

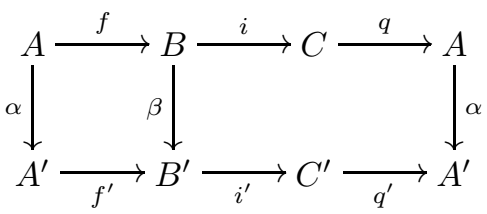

with exact rows can be completed to a morphism with exact mapping cone.

Suppose that the upper row in (6) is contractible and the lower row is quasiexact. Since $f^{\prime} \alpha=\beta f$ then $f^{\prime} \alpha q=0$; since $C$ is projective, there exists $\gamma^{\prime}: C \rightarrow C^{\prime}$ such that $q^{\prime} \gamma^{\prime}=\alpha q$. Let $(\Theta, \Phi, \Psi)$ be a contracting homotopy for the upper row. Then $\gamma=\gamma^{\prime}+\left(i^{\prime} \beta-\gamma^{\prime} i\right) \Phi$ completes (6) to a morphism of candidate triangles.

If the upper row in (6) is quasi-exact and the lower row is contractible then (6) can also be completed to a morphism. This can be shown directly, but it also follows from the previous case since we have a duality functor

$$
\operatorname{Hom}_{R}(-, R): \mathcal{F}(R) \stackrel{\sim}{\longrightarrow} \mathcal{F}(R)^{o p},
$$

which preserves contractible triangles and quasi-exact triangles. Here we use that $R$ is injective as an $R$-module, see Lam99, Example 3.12].

If the upper and the lower rows in (6) are $X_{2}$ and $Y_{2}$, respectively, then $\gamma=\beta+2 \delta$ extends (6) to a morphism of candidate triangles for any $\delta: X \rightarrow Y$.

This proves that any diagram like (6) with exact rows can be completed to a morphism $\varphi=(\alpha, \beta, \gamma)$. Now we have to check that the completion can be done in such a way that the mapping cone is exact. Suppose that the upper and the lower rows are $X_{2} \oplus T$ and $Y_{2} \oplus T^{\prime}$, respectively, with $T$ and $T^{\prime}$ contractible. The morphism $\varphi$ is given by a matrix of candidate triangle morphisms

$$
\varphi=\left(\begin{array}{ll}
\varphi_{11} & \varphi_{12} \\
\varphi_{21} & \varphi_{22}
\end{array}\right): X_{2} \oplus T \longrightarrow Y_{2} \oplus T^{\prime}
$$

where $\varphi_{i j}=\left(\alpha_{i j}, \beta_{i j}, \gamma_{i j}\right)$. Here $\varphi_{12}, \varphi_{21}$ and $\varphi_{22}$ are homotopic to 0 since either the source or the target is contractible, therefore the mapping cone of $\varphi$ is isomorphic to the mapping cone of

$$
\psi=\left(\begin{array}{cc}
\varphi_{11} & 0 \\
0 & 0
\end{array}\right): X_{2} \oplus T \longrightarrow Y_{2} \oplus T^{\prime},
$$

which is the direct sum of the mapping cone of $\varphi_{11}$ and two contractible triangles, $T^{\prime}$ and the translate of $T$. 
We can suppose that

$$
\alpha_{11}=\left(\begin{array}{lll}
1 & 0 & 0 \\
0 & 2 & 0 \\
0 & 0 & 0
\end{array}\right): X=L \oplus M \oplus N \longrightarrow L \oplus M \oplus P=Y .
$$

Moreover, as we have seen above we can take $\gamma_{11}=\beta_{11}+2 \delta$ for

$$
\delta=\left(\begin{array}{lll}
0 & 0 & 0 \\
0 & 1 & 0 \\
0 & 0 & 0
\end{array}\right): X=L \oplus M \oplus N \longrightarrow L \oplus M \oplus P=Y .
$$

We have $2 \beta_{11}=2 \alpha_{11}$, therefore $\beta_{11}=\alpha_{11}+2 \Phi$ for some $\Phi: X \rightarrow Y$. Now we observe that $(\delta, \Phi, 0)$ is a homotopy from $\varphi_{11}$ to $\zeta=\left(\alpha_{11}+2 \delta, \alpha_{11}+2 \delta, \alpha_{11}+2 \delta\right)$, so the mapping cone of $\varphi_{11}$ is isomorphic to the mapping cone of $\zeta$.

The mapping cone of $\zeta$ is clearly the direct sum of five candidate triangles, namely $M_{2}, N_{2}, M_{2}$ (once again), $P_{2}$, and the mapping cone of the identity $1: L_{2} \rightarrow L_{2}$, which is contractible. Therefore the mapping cone of $\zeta$ is exact, and also the mapping cone of $\varphi_{11}, \psi$ and $\varphi$.

It remains to show the uniqueness claim in Theorem [1. In any triangulation, all contractible candidate triangles are exact [Nee01, 1.3.8]. The triangle $X_{2}$ is a finite direct sum of copies of $R_{2}$. Hence every triangulation of $(\mathcal{F}(R), \operatorname{Id})$ which contains $R_{2}$ contains all the exact triangles which we considered above. Two triangulations with the same translation functor necessarily agree if one class of triangles is contained in the other, so there is only one triangulation in which $R_{2}$ is exact. This completes the proof.

Remark 7. The exact triangles in $\mathcal{F}(R)$ can be characterized more intrinsically as follows. Let $T$ be a quasi-exact triangle, which we can regard as a $\mathbb{Z} / 3$-graded chain complex of free $R$-modules with $H_{*}(T)=0$. As $T$ is free we have a short exact sequence

$$
2 T \hookrightarrow T \stackrel{2}{\rightarrow} 2 T,
$$

and the resulting long exact sequence in homology reduces to an isomorphism $\sigma: H_{*}(2 T) \rightarrow H_{*-1}(2 T)$. As the grading is 3 -periodic we can regard $\sigma^{3}$ as an automorphism of $H_{*}(2 T)$. We claim that $T$ is exact if and only if $\sigma^{3}=1$. One direction is straightforward: if $T$ is contractible then $H_{*}(2 T)=0$, and if $T=X_{2}$ then $H_{i}(2 T)=2 X$ for all $i$ and $\sigma$ is the identity. The converse is more fiddly and we will not go through the details. It would be nice to give a proof of Theorem 1 based directly on this definition of exactness, but we do not know how to do so.

Remark 8 . Let $k$ be a field of characteristic 2 . The same arguments as in the proof of Theorem 1 show that the category $\mathcal{F}\left(k[\varepsilon] / \varepsilon^{2}\right)$ of finitely generated free modules over the algebra $k[\varepsilon] / \varepsilon^{2}$ of dual numbers admits a triangulation with the identity translation functor and such that the diagram

$$
k[\varepsilon] / \varepsilon^{2} \stackrel{\varepsilon}{\longrightarrow} k[\varepsilon] / \varepsilon^{2} \stackrel{\varepsilon}{\longrightarrow} k[\varepsilon] / \varepsilon^{2} \stackrel{\varepsilon}{\longrightarrow} k[\varepsilon] / \varepsilon^{2}
$$

is an exact triangle. However, this triangulated category is both algebraic and topological, and hence, from our current perspective, less interesting.

Indeed $\mathcal{F}\left(k[\varepsilon] / \varepsilon^{2}\right)$ is an algebraic and topological triangulated category for any field $k$. The translation functor $\Sigma=\tau^{*}$ is the restriction of scalars along the $k$-algebra automorphism $\tau: k[\varepsilon] / \varepsilon^{2} \rightarrow k[\varepsilon] / \varepsilon^{2}$ with $\tau(\varepsilon)=-\varepsilon$, and

$$
k[\varepsilon] / \varepsilon^{2} \stackrel{\varepsilon}{\longrightarrow} k[\varepsilon] / \varepsilon^{2} \stackrel{\varepsilon}{\longrightarrow} k[\varepsilon] / \varepsilon^{2} \stackrel{\varepsilon}{\longrightarrow} \tau^{*} k[\varepsilon] / \varepsilon^{2}
$$


is an exact triangle. An algebraic model for this triangulated category was obtained by Keller in Kel05. Keller's model is a differerential graded (dg) $k$-category. Here we exhibit an alternative model, which is a $\operatorname{dg} k$-algebra $A$ such that $\mathcal{F}\left(k[\varepsilon] / \varepsilon^{2}\right)$ is exact equivalent to the category of compact objects in the derived category $\mathcal{D}(A)$ of dg (right) $A$-modules. This shows that $\mathcal{F}\left(k[\varepsilon] / \varepsilon^{2}\right)$ is both algebraic and topological.

Let $A=k\left\langle a, u, v, v^{-1}\right\rangle / I$ be the free graded $k$-algebra generated by $a, u, v$ and $v^{-1}$ in degrees $|a|=|u|=0$ and $|v|=-1$ modulo the two-sided homogeneous ideal $I$ generated by

$$
a^{2}, a u+u a+1, a v+v a \text { and } u v+v u .
$$

The differential $d: A \rightarrow A$ is determined by

$$
d(a)=u^{2} v, d(u)=0, \quad d(v)=0,
$$

and the Leibniz rule. The ungraded algebra $H_{0}(A)$ is isomorphic to the dual numbers $k[\varepsilon] / \varepsilon^{2}$, where $\varepsilon=[u]$ is the homology class of the cycle $u$. The graded algebra $H_{*}(A)$ is determined by this isomorphism since $[v]$ is a unit in degree -1 such that $\varepsilon \cdot[v]+[v] \cdot \varepsilon=0$.

We claim that the 0-dimensional homology functor

$$
H_{0}: \mathcal{D}^{c}(A) \longrightarrow \mathcal{F}\left(k[\varepsilon] / \varepsilon^{2}\right)
$$

is an equivalence of categories, where the left hand side is the full subcategory of those dg $A$-modules whose $H_{0}$ is finitely generated over $k[\varepsilon] / \varepsilon^{2}$.

Let $M$ be any $\operatorname{dg} A$-module and let $[x] \in H_{0}(M)$ be a homology class with $[x] \cdot \varepsilon=0$. We choose a representing cycle $x$ and an element $y$ with $d(y)=x u$; then the element $z=y u v-x a$ is a cycle with $x=z u-d(y a)$, so $[x]=[z] \cdot \varepsilon$ in homology. So every homology class which is annihilated by $\varepsilon$ is also divisible by $\varepsilon$, which proves that $H_{0}(M)$ is a free $k[\varepsilon] / \varepsilon^{2}$-module. Moreover, the translation functor in $\mathcal{D}(A)$ is the usual shift of complexes $M \mapsto M[1]$ and the natural isomorphism

$$
\tau^{*} H_{0}(M) \cong H_{0}(M[1])=H_{-1}(M)
$$

is given by $[x] \mapsto[x v]$.

The universal case of this is $M=A\{x, y\}$, the free graded right module over the underlying graded algebra of $A$ with $|x|=0$ and $|y|=1$. We can endow $M$ with a $\operatorname{dg} A$-module structure with $d(x)=0$ and $d(y)=x u$, so that $M$ is just the mapping cone of the chain map $A \stackrel{u}{\rightarrow} A$. The cycle $z=y u v-x a \in M$ gives a quasiisomorphism $A \rightarrow M$. Using this, we obtain an exact triangle

$$
A \stackrel{u}{\longrightarrow} A \stackrel{u}{\longrightarrow} A \stackrel{u v}{\longrightarrow} A[1]
$$

in $\mathcal{D}^{c}(A)$ which maps to the exact triangle (9). The rest of the proof that $H_{0}$ is an exact equivalence from $\mathcal{D}^{c}(A)$ to $\mathcal{F}\left(k[\varepsilon] / \varepsilon^{2}\right)$ is relatively straightforward, and we omit it.

We still owe the proof that the triangulated category $\mathcal{F}(R)$ does not admit nontrivial exact functors to or from a topological triangulated category. For this purpose we introduce two intrinsic properties that an object $A$ of a triangulated category may have.

A Hopf map for an object $A$ is a morphism $\eta: \Sigma A \rightarrow A$ which satisfies $2 \eta=0$ and such that for some (hence any) exact triangle

$$
A \stackrel{2}{\longrightarrow} A \stackrel{i}{\longrightarrow} C \stackrel{q}{\longrightarrow} \Sigma A
$$


we have $i \eta q=2 \cdot 1_{C}$. An object which admits a Hopf map will be termed hopfian. We note that the class of hopfian objects is closed under isomorphism, suspension and desuspension. If $F$ is an exact functor with natural isomorphism $\tau: \Sigma F \cong F \Sigma$ and $\eta: \Sigma A \rightarrow A$ a Hopf map for $A$, then the composite $F(\eta) \tau: \Sigma F(A) \longrightarrow F(A)$ is a Hopf map for $F(A)$.

We call an object $E$ exotic if there exists an exact triangle

$$
E \stackrel{2}{\longrightarrow} E \stackrel{2}{\longrightarrow} E \stackrel{h}{\longrightarrow} \Sigma E
$$

for some morphism $h: E \rightarrow \Sigma E$. We note that the class of exotic objects is closed under isomorphism, suspension and desuspension. Every exact functor takes exotic objects to exotic objects. Every object of the triangulated category $\mathcal{F}(R)$ of Theorem 1 is exotic.

We remark without proof that the morphism $h$ which makes (11) exact is unique and natural for morphisms between exotic objects. We show below that $h$ is of the form $h=2 \psi$ for an isomorphism $\psi: E \rightarrow \Sigma E$.

Remark 12. The integer 2 plays a special role in the definition of exotic objects, which ultimately comes from the sign which arises in the rotation of a triangle. In more detail, suppose that there is an exact triangle

$$
E \stackrel{n}{\longrightarrow} E \stackrel{n}{\longrightarrow} E \stackrel{h}{\longrightarrow} \Sigma E
$$

for some integer $n$. We claim that if $E$ is nonzero, then $n \equiv 2 \bmod 4$ and $4 \cdot 1_{E}=0$, so that the triangle (13) equals the 'exotic' triangle (11) with $n=2$. Indeed, we can find a morphism $\psi: E \rightarrow \Sigma E$ which makes the diagram

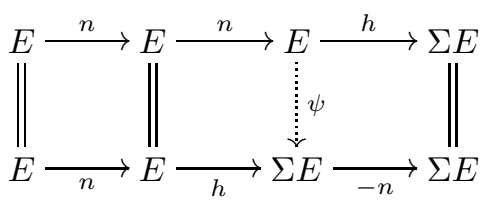

commute, and $\psi$ is an isomorphism. We have $n \psi=h=-n \psi$ which gives $2 n \psi=0$. Since $\psi$ is an isomorphism, this forces $2 n \cdot 1_{E}=0$. Exactness of (13) lets us choose a morphism $f: E \rightarrow E$ with $2 \cdot 1_{E}=n \cdot f$. But then $4 \cdot 1_{E}=n^{2} f^{2}=0$. So if $n$ is divisible by 4 , then $E=0$. If $n$ is odd, then $E$ is anhihilated by 4 and the odd number $n^{2}$, so also $E=0$.

Hopf maps are incompatible with the property of being exotic in the sense that these two classes of objects are orthogonal.

Proposition 14. Let $\mathcal{T}$ be a triangulated category, $A$ a hopfian object and $E$ an exotic object. Then the morphism groups $\mathcal{T}(A, E)$ and $\mathcal{T}(E, A)$ are trivial. In particular, every exotic and hopfian object is a zero object.

Proof. Let $\eta: \Sigma A \rightarrow A$ be a Hopf map. Given any morphism $f: E \rightarrow A$ there exists $g: E \rightarrow C$ such that $(f, f, g)$ is a morphism from (11) to (10), and hence if $=2 g=i \eta q g=i \eta(\Sigma f) h=i \eta(\Sigma f) 2 \psi=0$. Here we use the notation of Remark 12 for $n=2$ and the fact that $2 \eta=0$. Moreover, (10) is exact, so $f=2 f^{\prime}$ for some $f^{\prime}: E \rightarrow A$. This equation follows for any morphism $f: E \rightarrow A$, hence $f$ is divisible by any power of 2 , but $4 \cdot 1_{E}=0$, so $f=0$.

The proof of $\mathcal{T}(A, E)=0$ is similar. Alternatively, we can reduce this statement to the previous one by observing that the properties of being exotic and hopfian 
are self-dual. In other words, an object $E$ is exotic in a triangulated category $\mathcal{T}$ if and only if $E$ is exotic as an object of the opposite category $\mathcal{T}^{o p}$ with the opposite triangulation, and similarly for Hopf maps.

Proposition 15. Every object of a topological triangulated category is hopfian.

Proof. We can assume that the topological triangulated category is Ho $\mathcal{M}$ for a stable model category $\mathcal{M}$. We use that for every object $A$ of Ho $\mathcal{N}$ there exists an exact functor $F: \operatorname{Ho} \delta p \rightarrow$ Ho $\mathcal{M}$ from the stable homotopy category which takes the sphere spectrum to an object isomorphic to $A$. Here $\mathcal{S} p$ is the category of 'sequential spectra' of simplicial sets with the stable model structure of Bousfield and Friedlander [BF78, Sec. 2]. To construct $F$ we let $X$ be a cofibrant-fibrant object of the model category $\mathcal{M}$ which is isomorphic to $A$ in the homotopy category Ho $\mathcal{M}$. The universal property of the model category of spectra SS02, Thm. 5.1 (1)] provides a Quillen adjoint functor pair

$$
\mathcal{S} p \underset{\operatorname{Hom}(X,-)}{\stackrel{X \wedge}{\rightleftarrows}} \mathcal{M}
$$

whose left adjoint $X \wedge$ takes the sphere spectrum $S$ to $X$, up to isomorphism. The left derived functor of the left Quillen functor $X \wedge-: S p \rightarrow \mathcal{M}$ is exact and can serve as the required functor $F$.

Since exact functors preserve Hopf maps it thus suffices to treat the 'universal example', i.e., to exhibit a Hopf map for the sphere spectrum as an object of the stable homotopy category. The stable homotopy class $\eta: \Sigma S \rightarrow S$ of the Hopf map from the 3 -sphere to the 2 -sphere precisely has this property, hence the name. In more detail, we have an exact triangle

$$
S \stackrel{2 \cdot 1_{S}}{\longrightarrow} S \stackrel{i}{\longrightarrow} S / 2 \stackrel{q}{\longrightarrow} \Sigma S
$$

in the stable homotopy category, where $S / 2$ is the mod-2 Moore spectrum; then the morphism $2 \cdot 1_{S / 2}$ factors as $i \eta q$, and moreover $2 \eta=0$.

In topological triangulated categories, something a little stronger than Proposition 15 is true in that Hopf maps can be chosen naturally for all objects. However, we don't need this and so we omit the details. Now we can give the

Proof of Theorem Q Every object of the triangulated category $\mathcal{F}(R)$ is exotic and every object of a topological triangulated category is hopfian. So an exact functor from one type of triangulated category to the other hits objects which are both exotic and hopfian. But such objects are trivial by Proposition 14 .

Remark 16. The only special thing we use in the proof of Theorem 2 about topological triangulated categories is that therein every object has a Hopf map. Hopf maps can also be obtained from other kinds of structure that were proposed by different authors in order to 'enrich' or 'enhance' the notion of a triangulated category. So our argument also proves that the triangulated category $\mathcal{F}(R)$ of Theorem 1 does not admit such kinds of enrichments, and every exact functors to or from such enriched triangulated categories is trivial. For example, if $\mathcal{T}$ is an algebraic triangulated category, then for some (hence any) exact triangle (10) we have $2 \cdot 1_{C}=0$; so the zero map is a Hopf map.

Another example of such extra structure is the notion of a triangulated derivator, due to Grothendieck Gro90, and the closely related notions of a stable homotopy 
theory in the sense of Heller Hel88, Hel97. or a system of triangulated diagram categories in the sense of Franke Fra96. In each of these settings, the stable homotopy category is the underlying category of the free example on one generator (the sphere spectrum). We do not know a precise reference of this fact for triangulated derivators, but we refer to [Cis02, Cor. 4.19] for the 'unstable' (i.e., non-triangulated) analog. In Franke's setting the universal property is formulated as Theorem 4 of Fra96. These respective universal properties in the enhanced context provide, for every object $A$, an exact functor $\left(H_{o} \delta p\right)^{\mathrm{cp}} \rightarrow \mathcal{T}$ which takes the sphere spectrum $S$ to $A$, up to isomorphism. This functors sends the classical Hopf map for the sphere spectrum to a Hopf map for $A$.

Another kind of structure which underlies many triangulated categories is that of a stable infinity category as investigated by Lurie in Lur06. The appropriate universal property of the infinity category of spectra is established in [Lur06, Cor. 17.6], so again every object of the homotopy category of any stable, presentable infinity category has a Hopf map.

\section{REFERENCES}

[BF78] A. K. Bousfield and E. M. Friedlander, Homotopy theory of $\Gamma$-spaces, spectra, and bisimplicial sets, Geometric applications of homotopy theory (Proc. Conf., Evanston, Ill., 1977), II. Lecture Notes in Math., vol. 658, Springer, Berlin, 1978, pp. 80-130.

[Cis02] D.-C. Cisinski, Propriétés universelles et extensions de Kan dérivées, Preprint (2002).

[Coh46] I. S. Cohen, On the structure and ideal theory of complete local rings, Trans. Amer. Math. Soc. 59 (1946), 54-106.

[Fra96] J. Franke, Uniqueness theorems for certain triangulated categories possessing an Adams spectral sequence, $K$-theory Preprint Archives \#139 (1996). http://www.math.uiuc.edu/K-theory/

[GM03] S. I. Gelfand and Y. I. Manin, Methods of homological algebra, second ed., Springer Monographs in Mathematics, Springer-Verlag, Berlin, 2003.

[Gro90] A. Grothendieck, Dérivateurs, manuscript, around 1990, partially available from http://www.math.jussieu.fr/ maltsin/groth/Derivateurs.html

[Hel68] A. Heller, Stable homotopy categories, Bull. Amer. Math. Soc. 74 (1968), 28-63.

[Hel88] A. Heller, Homotopy theories, Mem. Amer. Math. Soc. 71 (1988), no. 383, vi+78 pp.

[Hel97] A. Heller, Stable homotopy theories and stabilization, J. Pure Appl. Algebra 115 (1997), 113-130.

[Hov99] M. Hovey, Model categories, Mathematical Surveys and Monographs, vol. 63, American Mathematical Society, Providence, RI, 1999.

[Kel05] B. Keller, On triangulated orbit categories, Doc. Math. 74 (2005), 551-581.

[Kel06] B. Keller, On differential graded categories, Proceedings of the International Congress of Mathematicians, Madrid, Spain, 2006, vol. II, European Mathematical Society, 2006, pp. 151-190.

[Lam99] T. Y. Lam, Lectures on modules and rings, Graduate Texts in Mathematics, 189. Springer-Verlag, New-York, 1999.

[Lur06] J. Lurie, Derived algebraic geometry I: Stable infinity categories. $\backslash$ protect \vrule width0pt \protect \href \{http://arxiv.org/abs/math/0608228\}\{math.CT/0608228\}

[Nee01] A. Neeman, Triangulated Categories, Annals of Mathematics Studies, vol. 148, Princeton University Press, Princeton, NJ, 2001.

[Sch06] S. Schwede, Algebraic versus topological triangulated categories, Extended notes of a talk given at the ICM 2006 Satellite Workshop on Triangulated Categories, Leeds, UK, http://www.math.uni-bonn.de/people/schwede/leeds.pdf, 2006.

[SS02] S. Schwede and B. Shipley, A uniqueness theorem for stable homotopy theory, Math. Z. 239 (2002), 803-828. 
Universitat de Barcelona, Departament d’Àlgebra i Geometria, Gran Via de les Corts Catalanes, 585, 08007 Barcelona, Spain

E-mail address: fmuro@ub.edu

Mathematisches Institut, Universität Bonn, Beringstr. 1, 53115 Bonn, Germany

E-mail address: schwede@math.uni-bonn.de

Department of Pure Mathematics, University of Sheffield, Hicks Building, Hounsfield ROAD, SHEFFIELD S3 7RH, UK

E-mail address: n.p.strickland@sheffield.ac.uk 